\title{
Motion characteristics of the vertebral segments with lumbar degenerative spondylolisthesis in elderly patients
}

\author{
Jun Miao $\cdot$ Shaobai Wang $\cdot$ Zongmiao Wan \\ Won Man Park · Qun Xia · Kirkham Wood • \\ Guoan Li
}

Received: 16 March 2012/Revised: 18 June 2012/ Accepted: 1 July 2012/Published online: 15 August 2012

(C) Springer-Verlag 2012

\begin{abstract}
Objective Although some studies have reported on the kinematics of the lumbar segments with degenerative spondylolisthesis (DS), few data have been reported on the in vivo 6 degree-of-freedom kinematics of different anatomical structures of the diseased levels under physiological loading conditions. This research is to study the in vivo motion characteristics of the lumbar vertebral segments with L4 DS during weight-bearing activities.

Methods Nine asymptomatic volunteers (mean age 54.4) and 9 patients with L4 DS (mean age 73.4) were included. Vertebral kinematics was obtained using a combined MRI/ $\mathrm{CT}$ and dual fluoroscopic imaging technique. During functional postures (supine, standing upright, flexion, and extension), disc heights, vertebral motion patterns and instability were compared between the two groups.
\end{abstract}

J. Miao $\cdot$ S. Wang $\cdot$ Z. Wan $\cdot$ W. M. Park $\cdot$ K. Wood

G. Li (ه)

Bioengineering Lab, Department of Orthopaedic Surgery,

Massachussets General Hospital/Harvard Medical School,

55 Fruit St., GRJ 1215, Boston, MA 02114, USA

e-mail: gli1@partner.org

J. Miao - Q. Xia

Department of Spinal Surgery, Tianjin Hospital, Tianjin, China

S. Wang

Department of Mechanical Engineering,

Massachusetts Institute of Technology, Cambridge, MA, USA

Z. Wan

Second Xiangya Hospital of Central South University,

Changsha, China

W. M. Park

Department of Mechanical Engineering, Kyung Hee University, Yongin, Korea
Results Although anterior disc heights were smaller in the DS group than in the normal group, the differences were only significant at standing upright. Posterior disc heights were significantly smaller in DS group than in the normal group under all postures. Different vertebral motion patterns were observed in the DS group, especially in the left-right and cranial-caudal directions during flexion and extension of the body. However, the range of motions of the both groups were much less than the reported criteria of lumbar spinal instability.

Conclusion The study showed that lumbar vertebra with DS has disordered motion patterns. DS did not necessary result in vertebral instability. A restabilization process may have occurred and surgical treatment should be planned accordingly.

Keywords Lumbar spine - Degenerative spondylolisthesis · Vertebral kinematics . Disc degeneration $\cdot$ Spinal instability

\section{Introduction}

Lumbar degenerative spondylolisthesis (DS) is the forward slippage of a superior lumbar vertebra relative to the inferior vertebra due to degeneration, with the pars interarticularis intact. Lumbar DS was recognized as a common condition in elder individuals [1], which frequently causes mechanical low back pain, radicular lower limb pain and neurogenic claudication [2]. A large series study ( $n=3,259$ outpatients with low back pain) showed that the overall incidence of DS is $8.7 \%$, and the predominant segment and gender are L4-5 and female [1]. Although eight decades has passed since Junghanns firstly described the condition in 1930, the etiology, pathogenesis, and treatment of DS are still controversial [2]. 
Most previous studies investigated the relationship between DS and morphological changes in the lumbar spine structures such as the facet joint and the intervertebral disc. Some studies reported that increased sagittal orientation of the facet joint and the pedicle-facet angle, $\mathrm{W}$-shaped facet joint, horizontalization of the lamina, and facet joint osteoarthritis are predisposing factors of DS [1-5]. Others reported that disc degeneration was the main reason of DS, as the disc height loss not only raises the facet joint pressure that leads to arthritic remodeling [6], but also produces sagittal plane instability that leads to DS [7].

In addition to morphologic changes, altered kinematics is assumed to be another important factor that is related to DS and its surgical treatment $[8,9]$. Due to slippage, kinematics of the vertebrae with DS is altered, which may lead to a series of pathological processes and clinical symptoms. To reveal pathogenesis, explain clinical symptoms and decide treatment methods, kinematics of DS has been studied using a variety of techniques including lateral flexion-extension radiographs [10], biplanar radiographs [11], cineradiography [12, 13], open MRI [14, 15] and, etc. However, most of these studies focused on the anterior-posterior motion of the vertebral bodies. Since both anterior disc and posterior facet joints may play important roles during the process of DS, it is important to study the kinematics of each part of the vertebrae to understand pathogenesis, explain clinical symptoms, and select treatment methods. However, there are few data reported on the in vivo 6 degree-of-freedom (6DOF) kinematics of different anatomic structures of the diseased levels under physiological loading conditions.

We have developed a combined dual fluoroscopic and $\mathrm{MR} / \mathrm{CT}$ imaging system (DFIS) to investigate the in vivo lumbar spine kinematics [16]. Using this technique, the 6 DOF kinematics of various structures of the vertebrae can be accurately measured when the subjects are at different physiological positions [17]. In this study, we measured the in vivo kinematics of the L4-5 vertebrae with DS in a cohort of elderly patients, and compared the results with those of a group of asymptomatic normal elder subjects.

\section{Materials and methods}

\section{Study populations}

Approval of the experimental design by the authors' Institutional Review Board was obtained prior to the initiation of the study. A written consent was obtained from each subject prior to the study. Nine patients with diagnosis of L4-5 DS (3 males and 6 females) were recruited from a single academic center. The patients had a mean age of 73.6 years (range 52-87 years). All patients vertebrae slippage were grade I by Meyerding classification method that categorizes severity of the slippage based on the percentage of translation of the upper vertebral body over the lower one. Nine asymptomatic subjects with a mean age of 54.4 years (range 50-60 years, 4 males and 5 females) were recruited for this study. The subjects were evaluated for the absence of low back pain and other spinal disorders.

MRI/CT-based three-dimensional geometry model of vertebrae

All subjects were scanned in a supine, relaxed position. Two patients with DS and nine normal subjects were scanned using a Siemens Medical Solutions MAGNETOM Trio 3-T MRI scanner with a spine surface coil and a T2weighted fat-suppressed 3-D spoiled gradient recalled sequence. Sagittal images with thickness of $1.5 \mathrm{~mm}$ and resolution of $512 \times 512$ pixels were obtained. Seven patients with DS were scanned in a General Electric LightSpeed Pro16 CT scanner. Axial plane images with thickness of $0.625 \mathrm{~mm}$ and resolution of $512 \times 512$ pixels were obtained. The images of the spinal segments were then imported into a modeling software (Rhinoceros ${ }^{\circledR}$ Robert McNeel \& Associates, Seattle, Washington) to construct 3-D geometric vertebrae models of L4 and L5. Models created from CT or MRI images have no significant difference in geometry and thus have similar accuracy in matching results according to our previous study [18].

\section{Dual fluoroscopic imaging}

The lumbar spine was imaged using a dual fluoroscopic system (BV Pulsera; Philips, Bothell, Washington). The subjects were asked to stand and position their lumbar spines within the views of both fluoroscopes and actively move to different postures: standing upright, maximum lumbar flexion, maximal lumbar extension. The subjects were also asked to self-limit the hip rotation and focus on lumbar motion. In patients with DS, certain maximum positions may cause pain. But the imaging time was about $1 \mathrm{~s}$ so that all patients can finish the study. Images were taken as described by previous paper [16].

Reproduction of lumbar spine kinematics under in vivo weight bearing

The geometry of the dual-orthogonal fluoroscopic system was created in the modeling software [16]. The lumbar vertebrae models were introduced into the virtual system and were independently moved and rotated until their silhouettes matched with those captured on the two orthogonal fluoroscopic images. Thus, the positions of the lumbar vertebrae during in vivo weight-bearing activities were reproduced. 


\section{Creation of coordinate system}

Right-hand Cartesian coordinate systems were created to quantify the $6 \mathrm{DOF}$ motions of several representative locations on L4 and L5 vertebrae (Fig. 1). For L4, five points were selected as the origin of coordinate systems: the midpoints of the anterior and posterior rim of inferior endplate, the center of the left and right inferior facet joints, and the center of the spinous process. Similarly for L5, five corresponding points were selected as the origin of the coordinate systems: the midpoints of anterior and posterior rim of superior endplate, the center of the left and right superior facet joints, and the center of the spinous process. The $x$ axis was set perpendicular to the sagittal plane and parallel to the superior endplate of L5 to
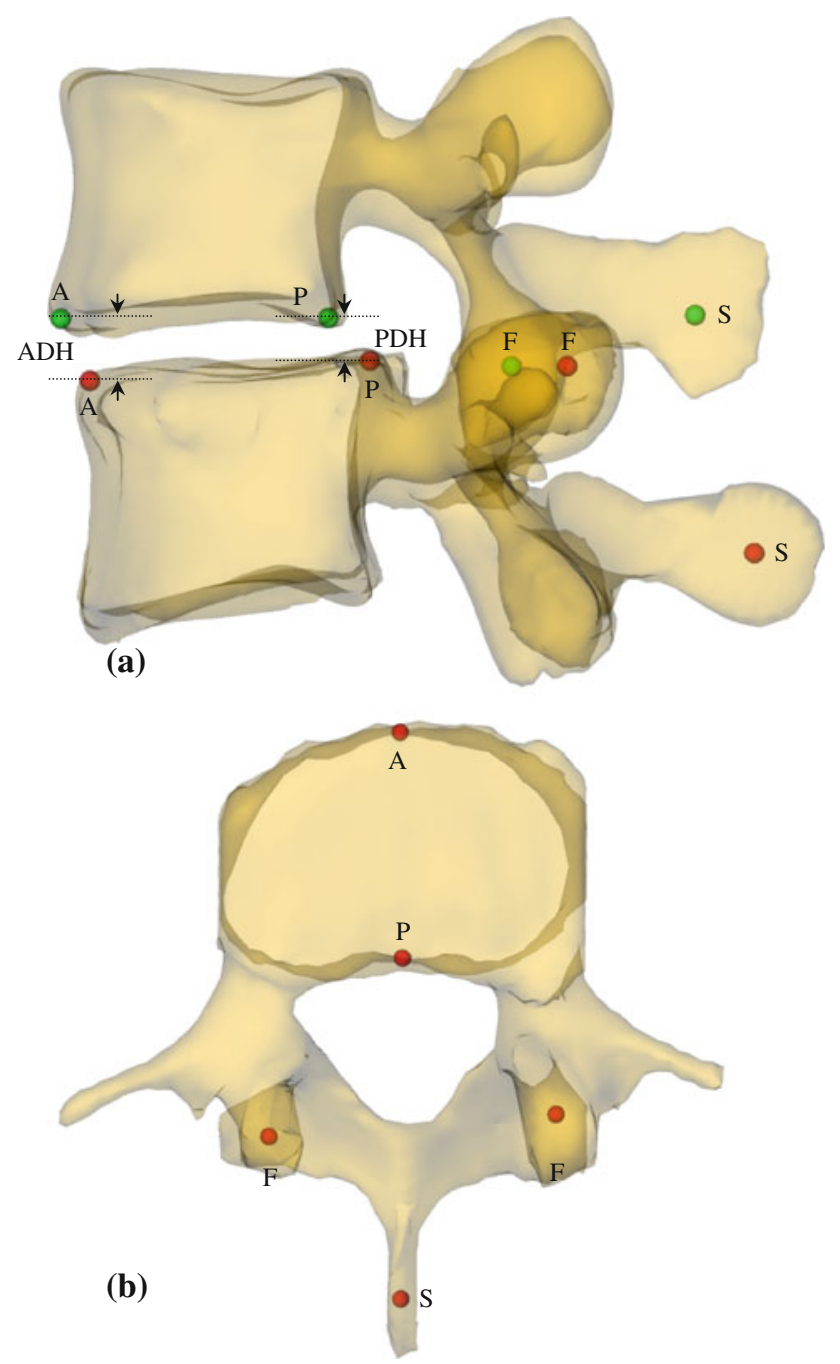

Fig. 1 Origins of the coordinate systems: a lateral view, b superior view, $A$ anterior rim of the endplate, $P$ posterior rim of the endplate, $F$ center of the facet joint, $S$ center of the spinous process; measurement of the disk height: $A D H$ anterior disk height, $P D H$ posterior disk height represent the left-right direction. The $y$ axis was set in sagittal plane parallel to the superior endplate of L5 to represent the anterior-posterior direction. The $z$ axis was perpendicular to the transverse plane to represent the cranial-caudal direction.

\section{Measurements of variables}

By measuring the corresponding proximal vertebral coordinate system in the distal coordinate system, motions of the disc, the facets and the spinous process were calculated at standing upright, flexion and extension with respect to relaxed supine position in 6DOF. Results were presented in left-right, anterior-posterior and cranial-caudal translations and rotations of these structures. In addition, anterior disc height $(\mathrm{ADH})$ and posterior disc height $(\mathrm{PDH})$ were, respectively, retrieved by measuring the perpendicular distance between the two opposite points of the endplate rims (Fig. 1). Stability was evaluated by comparing the $\mathrm{ROM}$ of flexion-extension.

\section{Statistical analysis}

The continuous variables were measured as mean $\pm \mathrm{SD}$ and one-way ANOVA test was used to examine betweengroup differences. All analyses were performed using the Statistical Package for the Social Sciences (SPSS for Windows, release 19.0, IBM) and the significant level of $P$ was set at 0.05 .

\section{Results}

In all postures, both $\mathrm{ADH}$ and $\mathrm{PDH}$ were shorter in the DS group than in the normal group (Table 1). PDH were significantly shorter in all postures $(p<0.05)$, while ADH were significantly shorter only during standing upright when compared the DS group $(6.93 \pm 3.23 \mathrm{~mm})$ to the normal group $(10.27 \pm 2.28 \mathrm{~mm})(p<0.05)$.

Motions of L4 with respect to L5 were plotted with supine posture as zero reference (Figs. 2, 3, 4). The horizontal axes were drawn to scale to reflect the averaged distances between various structures of the vertebra and marked on the figures. The vertical axes represent the range of motions (ROMs) of L4 under the other three postures relative to supine posture.

Small left-right translations (about $1 \mathrm{~mm}$ ) of different vertebral structures were observed in both groups (Fig. 2). In the normal group, a clear left-right swing pattern of the cranial vertebra with respect to the caudal vertebra was observed. During extension, anterior rim of the cranial vertebral body swing to the left and its spinous process swing to the right. During flexion, anterior rim of the 
Table 1 Disc height under different postures (mean $\pm \mathrm{SD}, n=9$ )

\begin{tabular}{llrrr}
\hline Postures & Disc height & Normal $(\mathrm{mm})$ & DS $(\mathrm{mm})$ & $p$ \\
\hline Supine & ADH & $10.70 \pm 1.90$ & $8.69 \pm 3.84$ & $3.99 \pm 1.84$ \\
& PDH & $6.35 \pm 2.10$ & 0.18 \\
Stand upright & ADH & $10.27 \pm 2.28$ & $0.93 \pm 3.23$ & 0.02 \\
& PDH & $6.07 \pm 1.97$ & $3.31 \pm 1.68$ & 0.01 \\
Extension & ADH & $10.91 \pm 2.13$ & $3.04 \pm 1.83$ & 0.10 \\
& PDH & $5.94 \pm 2.31$ & $6.50 \pm 3.41$ & 0.01 \\
Flexion & ADH & $9.20 \pm 2.64$ & $3.65 \pm 1.86$
\end{tabular}

$A D H$ anterior disk height, $P D H$ posterior disk height

Fig. 2 Motion of the vertebrae in left-right direction

Fig. 3 Motion of the vertebrae in anterior-posterior direction

Fig. 4 Motion of the vertebrae in cranial-caudal direction
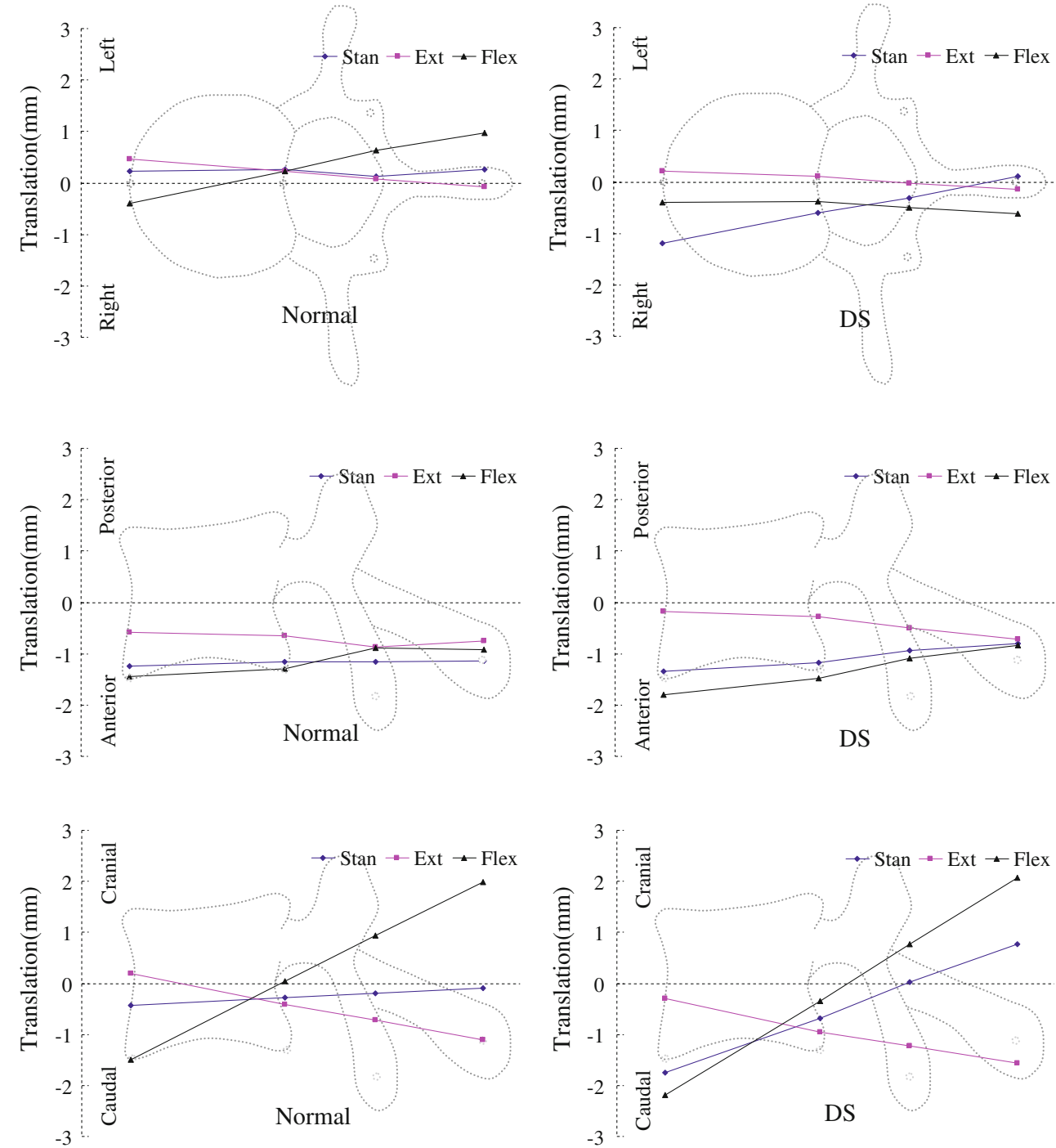

\subsection{8}

.02

0.02

0.01

0.10 0.01 0.02 
Table 2 ROM of extension to flexion (mean $\pm \mathrm{SD}, n=9$ )

\begin{tabular}{lllll}
\hline Direction & Structure & Normal $(\mathrm{mm})$ & DS $(\mathrm{mm})$ & $p$ \\
\hline Left-right & $\mathrm{A}$ & $2.16 \pm 1.51$ & $1.48 \pm 1.70$ & 0.38 \\
& $\mathrm{P}$ & $1.04 \pm 1.05$ & $1.79 \pm 1.59$ & 0.25 \\
& $\mathrm{~F}$ & $1.43 \pm 0.57$ & $2.07 \pm 1.28$ & 0.19 \\
Anterior-posterior & $\mathrm{S}$ & $2.01 \pm 1.36$ & $2.45 \pm 1.23$ & 0.48 \\
& $\mathrm{~A}$ & $1.19 \pm 0.65$ & $1.84 \pm 0.62$ & 0.04 \\
& $\mathrm{P}$ & $0.94 \pm 0.69$ & $1.59 \pm 0.22$ & 0.02 \\
& $\mathrm{~F}$ & $0.99 \pm 0.71$ & $1.30 \pm 0.59$ & 0.33 \\
Cranial-caudal & $\mathrm{S}$ & $0.81 \pm 0.80$ & $1.39 \pm 0.93$ & 0.18 \\
& $\mathrm{~A}$ & $1.70 \pm 0.93$ & $1.89 \pm 1.03$ & 0.70 \\
& $\mathrm{P}$ & $0.97 \pm 0.63$ & $1.14 \pm 0.89$ & 0.65 \\
& $\mathrm{~F}$ & $1.96 \pm 1.62$ & $2.40 \pm 1.74$ & 0.59 \\
& $\mathrm{~S}$ & $3.45 \pm 2.58$ & $4.04 \pm 2.87$ & 0.65 \\
\hline
\end{tabular}

$A$ anterior rim of the endplate, $P$ posterior rim of the endplate, $F$ center of the facet joint, $S$ center of the spinous process

in the DS group, there were significantly larger compressions of the anterior part of the vertebrae than the posterior spinous process. Overall, each part of the vertebrae of the DS group tended to have larger ROM during flexionextension, compared with that of the normal group. Significantly larger anterior-posterior translations were found in the DS group at the anterior and posterior of the vertebrae $(1.84 \pm 0.62 \mathrm{~mm}$ DS compared to $1.19 \pm 0.65 \mathrm{~mm}$ normal, and $1.59 \pm 0.22 \mathrm{DS}$ compared to $0.94 \pm 0.69 \mathrm{~mm}$ normal, respectively) (Table 2). Subprocess of stand extension and stand flexion were further analyzed. Subprocess results showed that there were no significant differences between the two groups during the subprocesses of stand extension and stand flexion.

\section{Discussion}

In vivo motion characteristics of the vertebral segments with DS are difficult to quantify in 6DOF and few data has been reported in literature. In this study, we used a combined MRI/CT and dual fluoroscopic image system to determine the disc heights, motion patterns and ROMs of the vertebral segment with DS in living patients under supine, standing upright, flexion and extension and compared the data to those of normal subjects. The combined imaging technique was able to investigate all 6DOF motion with sub-millimeter/-degree accuracy, which is particularly useful in study patients since the sagittal motion alone may not fully describe the deformity and abnormal motion associated with spinal diseases. We found that disc heights under the four postures were shorter in DS group compared with normal group. We also observed different motion patterns between the two groups during flexion and extension. However, we did not find any statistically significant difference in the ROMs between the two groups.

Association between intervertebral disc degeneration and DS remained controversial. Decreased intervertebral disc height, or narrowing was usually considered as a degenerative indicator $[7,19]$. Some studies indicated that intervertebral disc narrowing had no significant relationship with DS [5, 20]. Kalichman [5] found that age, sex and facet joint osteoarthritis, but not intervertebral disc narrowing, were significantly associated with DS in a crosssectional study. Vogt [20] found that anterolisthesis was not associated with the disc height among elder African American women in a lateral radiographs study. Some other studies, however, revealed that disc degeneration was associated with DS to a varying degree [21]. Disc degeneration was regarded as an initial event, which leads to the decrease of the disc height and then segmental instability, leading to slippage of the vertebrae [2, 7]. Chen [7] found that middle aged women with DS had decreased overall disc height and decreased ADH was an independent predictor of DS in a radiographic study. Fujiwara [22] found that anterior translational instability was positively associated with disc degeneration and facet joint osteoarthritis in a consecutive patients (mean age 46 years) using lumbar MRI and functional radiograph. In our data, we also observed that the ADH were smaller in the DS group than in the normal group but the differences were only significant at standing upright and the PDH was significantly reduced in all the four postures. The changes of disc heights in DS group indicated that the affected disc had increased vertical laxity and decreased elasticity [8, 23]. Our data indicated that disc degeneration had some relationship with DS.

Although various radiographic techniques have been used to study lumbar kinematics, few data have been reported on the in vivo $6 \mathrm{DOF}$ lumbar kinematics with DS during functional movement. Takayanagi [12] studied the motion patterns in asymptomatic volunteers and symptomatic patients with L4-5 DS in sitting postures and found that lumbar spine with DS presented disordered motion patterns and segmental instability. Okawa [13] reported that patients with DS showed disordered motion patterns while subject bent forward from a standing neutral position and then returned to the original position. In this study, the kinematics of both anterior and posterior structures of the slipped vertebrae was evaluated and different gross motion patterns from normal vertebrae were observed, which agreed with the existing literatures.

It is interesting to note that in the transverse plane, the normal vertebrae had a side to side swing motion centered at the posterior rim of vertebral body, while the slipped vertebrae had a disordered swing motion without a distinct pattern. The disordered movement of the slipped vertebrae 
maybe resulted from asymmetric degeneration and proliferation of the vertebral body, intervertebral disc and facet joints [24]. Although there was no statistical significance, the DS group tended to have larger translations, which revealed that the disc inferior to the slipped vertebrae in the DS group exhibit hypermobility. As shown in Fig. 2, the facet joints in the DS group had increased motion than the normal group, indicating that the facet joint of the slipped segment has increased laxity. Previous studies have shown that facet joints were more sagittally orientated in patients with DS and more coronal orientated in normal subjects [25], which implied that the facet joints in the DS group may be less efficient in limiting anterior-posterior translation of the slipped vertebrae.

In the cranial-caudal direction in the sagittal plane, the slipped vertebrae had different motion patterns from supine to standing upright compared to normal vertebrae. There were significantly larger compression of the anterior part of the vertebrae with DS compared to normal, while their posterior spinous processes were at slight tension, i.e., during flexion and extension, the posterior structures, the facet joints and the spinous process tend to "open wider" in the DS group than in the normal group (Fig. 4). This may be caused by the decreased disc height and elasticity of the affected disc as mentioned above. This motion may overstrain posterior spinal muscles for a longer period and ultimately leading to muscular injuries and fatty infiltration as hypothesized in the literature [26].

Instability is an important factor in explaining clinical symptoms and determining the surgical method, such as decompression without fusion, posterolateral fusion with or without instrumentation and anterior lumbar interbody fusion [2]. Whether DS leads to instability was controversial in literature [2]. DS was conventionally considered to be instable [2], but some papers have reported that there was no evidence that the ROMs of the vertebrae with DS were increased compared with normal vertebrae [2, 14]. McGregor [14] investigated the kinematics of flexion and extension in patients with degenerative and isthmic spondylolisthesis using an open MRI and did not find instability. Instability was usually determined from the 2D ROMs of the vertebrae using 2D extension-flexion lateral radiographs. Several radiographic diagnostic criteria have been proposed for lumbar spinal instability and the reported criteria had different cutoff value for different author, such as 3, 4 or $5 \mathrm{~mm}$ for translational motion [27]. More recently, Hasegewa presented a new intraoperative measurement system, with displacement transducer and load cell fixed to the spinous process to measure the force-displacement behavior during flexionextension. They proposed to use initial flexion stiffness as criteria for access instability. Widely distributed flexion stiffness was found in segments with DS and suggested that DS was not always unstable [28]. In the present study, we studied the 6DOF ROMs of the vertebrae in 3D spaces (Table 2). The ROM of DS in the current study was much less than the reported criteria of instability, which indicated that there was no obvious instability in the current DS group. Kirkaldy-Willis [29] subdivided the lumbar degenerative process into three stages: temporary dysfunction, unstable and restabilization stage. Using this classification, the presence of DS does not necessarily mean instability when restabilization occurred [30]. The reason may be that the ages of our patients were relatively high and the degeneration of lumbar had entered into the third stage of restabilization.

There were some limitations in the present study. First, the study only focused on the elder patient population. Further investigations are necessary before extending the results to general patient population of DS. The mean age of our healthy patients is smaller than that of the DS patients, since age of 40-60 is the eldest healthy population that we can get without severe degenerative changes in the lumbar spine, which may affect the lumbar motion characteristics. Second, the sample size is relatively small in the present study, mainly due to the difficulties in patient recruitment and follow-up of the elder population. However, we were still able to observe significant differences in motion characteristics between the two groups. With more subjects enrolled in future, only few marginal differences between the two groups, e.g., ADH at flexion $(p=0.08$, $72 \%$ power) might turn out to be significant. Third, only flexion and extension were examined in the current study, lateral bending and torsion as well as other activities should be investigated in future studies.

This study compared the vertebral kinematics of the patients with DS to normal subjects during weight bearing, standing upright, flexion, and extension. DS subjects had decreased disc height under different postures, indicating that the disc degeneration plays a role during the process of DS. Significantly different motion patterns of different vertebral structures, including the disc, facet and spinous process have also been observed. However, non-significant differences in ROMs were observed between the DS and normal subjects. The results suggested a restabilization of the vertebrae may have occurred in the elderly patients and surgical treatment should be planned accordingly.

Acknowledgments This study was supported by NIH (R21AR057 989) and China scholarship council.

Conflict of interest None.

\section{References}

1. Iguchi T, Wakami T, Kurihara A et al (2002) Lumbar multilevel degenerative spondylolisthesis: radiological evaluation and factors related to anterolisthesis and retrolisthesis. J Spinal Disord Tech 15:93-99 
2. Sengupta DK, Herkowitz HN (2005) Degenerative spondylolisthesis: review of current trends and controversies. Spine 15:S71S81

3. Berlemann U, Jeszenszky DJ, Bühler DW et al (1999) The role of lumbar lordosis, vertebral end-plate inclination, disc height, and facet orientation in degenerative spondylolisthesis. J Spinal Disord 12:68-73

4. Nagaosa Y, Kikuchi S, Hasue M et al (1998) Pathoanatomic mechanisms of degenerative spondylolisthesis. A radiographic study. Spine 23:1447-1451

5. Kalichman L, Hunter DJ, Kim DH et al (2009) Association between disc degeneration and degenerative spondylolisthesis? Pilot study. J Back Musculoskelet Rehabil 22:21-25

6. Varlotta GP, Lefkowitz TR, Schweitzer M et al (2011) The lumbar facet joint: a review of current knowledge: part 1: anatomy, biomechanics, and grading. Skeletal Radiol 40:13-23

7. Chen IR, Wei TS (2009) Disc height and lumbar index as independent predictors of degenerative spondylolisthesis in middleaged women with low back pain. Spine 34:1402-1409

8. Luk KD, Chow DH, Holmes A (2003) Vertical instability in spondylolisthesis: a traction radiographic assessment technique and the principle of management. Spine 28:819-827

9. Hong SW, Lee HY, Kim KH et al (2010) Interspinous ligamentoplasty in the treatment of degenerative spondylolisthesis: midterm clinical results. J Neurosurg Spine 13:27-35

10. Cho BY, Murovic JA, Park J (2009) Imaging correlation of the degree of degenerative L4-5 spondylolisthesis with the corresponding amount of facet fluid. J Neurosurg Spine 11:614-619

11. Pearcy M, Shepherd J (1985) Is there instability in spondylolisthesis? Spine 10:175-177

12. Takayanagi K, Takahashi K, Yamagata M et al (2001) Using cineradiography for continuous dynamic-motion analysis of the lumbar spine. Spine 26:1858-1865

13. Okawa A, Shinomiya K, Komori H et al (1998) Dynamic motion study of the whole lumbar spine by videofluoroscopy. Spine 23:1743-1749

14. McGregor AH, Anderton L, Gedroyc WM et al (2002) The use of interventional open MRI to assess the kinematics of the lumbar spine in patients with spondylolisthesis. Spine 27:1582-1586

15. Huang KY, Lin RM, Lee YL et al (2009) Factors affecting disability and physical function in degenerative lumbar spondylolisthesis of L4-5: evaluation with axially loaded MRI. Eur Spine J 18:1851-1857
16. Li G, Wang S, Passias P et al (2009) Segmental in vivo vertebral motion during functional human lumbar spine activities. Eur Spine J 18:1013-1021

17. Xia Q, Wang S, Kozanek M et al (2010) In vivo motion characteristics of lumbar vertebrae in sagittal and transverse planes. J Biomech 43:1905-1909

18. Wang S, Passias P, Li G, Wood K (2008) Measurement of vertebral kinematics using noninvasive image matching methodvalidation and application. Spine 33:E355-E361

19. Pfirmann CW, Metzdorf A, Elfering A et al (2006) Effect of aging and degeneration on disc volume and shape: a quantitative study in asymptomatic volunteers. J Orthop Res 24:1086-1094

20. Vogt MT, Rubin DA, Palermo L et al (2003) Lumbar spine listhesis in older African American women. Spine J 3:255-261

21. Bolesta MJ, Bohlman HH (1989) Degenerative spondylolisthesis. Instr Course Lect 38:157-165

22. Fujiwara A, Tamai K, An HS et al (2000) The relationship between disc degeneration, facet joint osteoarthritis, and stability of the degenerative lumbar spine. J Spinal Disord 13:444-450

23. Chagnon A, Aubin CE, Villemure I (2010) Biomechanical influence of disk properties on the load transfer of healthy and degenerated disks using a poroelastic finite element model. J Biomech Eng 132:111006

24. Dai L, Jia L (1996) Role of facet asymmetry in lumbar spine disorders. Acta Orthop Belg 62:90-93

25. Toyone T, Ozawa T, Kamikawa K et al (2009) Facet joint orientation difference between cephalad and caudad portions: a possible cause of degenerative spondylolisthesis. Spine 34:22592262

26. Ramsbacher J, Theallier-Janko A, Stoltenburg-Didinger G et al (2001) Ultrastructural changes in paravertebral muscles associated with degenerative spondylolisthesis. Spine 26:2180-2185

27. Alqarni AM, Schneiders AG, Hendrick PA (2011) Clinical tests to diagnose lumbar segmental instability: a systematic review. J Orthop Sports Phys Ther 41:130-140

28. Hasegewa K, Kitahara K, Hara T, Takano K, Shimoda H (2009) Biomechanical evaluation of segmental instability in degenerative lumbar spondylolisthesis. Eur Spine J 18(4):465-470

29. Kirkaldy-Willis WH, Farfan HF (1982) Instability of the lumbar spine. Clin Orthop Relat Res 165:110-123

30. Nizard RS, Wybier M, Laredo JD (2001) Radiologic assessment of lumbar intervertebral instability and degenerative spondylolisthesis. Radiol Clin North Am 39:55-71 (v-vi) 